\title{
A Two-Stage Framework for Polygon Retrieval Using Minimum Circular Error Bound
}

\author{
Lun Hsing Tung and Irwin King \\ \{lhtung, king\}@cse.cuhk.edu.hk \\ Department of Computer Science and Engineering, \\ The Chinese University of Hong Kong, \\ Shatin, New Territories, Hong Kong
}

\begin{abstract}
We have proposed a two-stage framework for polygon retrieval $[12,11]$ which incorporates both qualitative and quantitative measures of polygons in the first and second stage respectively. In this paper, we introduce an extension to our two-stage framework. We propose a new polygon matching technique using Circular Error Bound and describe how this technique works under translation and scaling of polygons. Base on this technique, we propose a new translation invariant similarity measure for polygons named Minimum Circular Error Bound, which can be used in the second stage of the two-stage framework. We compare the Minimum Circular Error Bound method with the Hausdorff Distance method and demonstrate the advantages of our method.
\end{abstract}

\section{Introduction}

Shape matching and measuring similarity between shapes are important issues in pattern recognition. They can be applied in many applications, such as providing query-by-shape facility in image database systems and constructing hand writing recognition systems. In this paper, we focus on the matching of polygonal shapes instead of arbitrary shapes, since shapes are often represented by polygons and polygon approximation of shapes is acceptable in many applications.

In $[12,11]$, we proposed a two-stage framework for polygon retrieval in image databases. The first stage of the framework uses the Binary Shape Descriptor (BSD) [2] technique to perform polygon classification and prune the search space in order to speed up query processing. The second stage of the framework uses any available polygon similarity measuring technique for quantitative measurement of the similarity between polygons. We proposed the Multi-Resolution Area Matching (MRAM) technique in $[12,11]$ as the technique to be incorporated at the second stage. In this paper, we propose an extension to the two-stage framework which allows systematic control on the degree of search space pruning.

Considerable works have been carried out on the polygon matching problem. Most of these researches extract features from polygons and use these features as similarity measure $[9,6,4,10]$. However, the similarity ranking of polygons produced by these methods may not coincide with human perception. In this paper, we propose a polygon matching technique using Circular Error Bound 
(CEB) which is based on an intuitive human concept of polygon resemblance. Using the same idea, we propose a polygon similarity measure named Minimum Circular Error Bound (MCEB) which produces polygon rankings resembling human rankings. The MCEB method can be used at the second stage of the two-stage framework for shape matching.

The two-stage framework is implemented in the Montage image database system [8] currently under development at the Chinese University of Hong Kong. The Montage system is an image database system designed for the fashion, textile, and clothing industry in Hong Kong. It supports feature based retrieval by color histogram, color sketch, shape, and texture.

This paper is organized as follows. We present the extension to the two-stage framework in Section 2. We propose the polygon matching technique using Circular Error Bound in Section 3. In Section 4, we present the Minimum Circular Error Bound similarity measure for polygons. The experimental results of our work will be discussed in Section 5. Conclusion is made in Section 6 .

\section{Extension to the Two-Stage Framework}

The two-stage framework approach we proposed in $[12,11]$ may fail to produce good matching results because of the first stage filtering mechanism. In Figure 1, there are three polygons named $P, Q$, and $R$. Using polygon $P$ as the target polygon, the proposed method will not be able to produce the result that polygon $Q$ is more similar to polygon $P$ than polygon $R$ does. It is because polygon $Q$ is not in the equivalent class as polygon $P$ so it will not be selected at the first stage. On the other hand, polygon $R$ is in the same equivalent class as polygon $P$ so it is selected for the second stage matching. Yet, polygon $Q$ appears to be more similar to polygon $P$ than polygon $R$ to polygon $P$.

To tackle this problem, we propose the following extension to the first stage of the two-stage framework. When a query is initiated, the SBSD of the target polygon is computed. All polygons inside the database having a SBSD within a user specified Hamming Distance to the SBSD of the target polygon are selected for the second stage processing. For example, if user specifies a Hamming Distance of 1, both polygon $Q$ and polygon $R$ in Figure 1 will be selected for second stage matching so polygon $Q$ will have the chance to be compared with polygon $P$. Whether polygon $Q$ is said to be more similar to polygon $P$ or not will still depends on the polygon similarity measuring method used at the second stage.

This extension provides a systematic way for controlling the degree of pruning database entries. Small Hamming Distance value has larger pruning effect but with higher risk of producing worse matching results. On the other hand, large Hamming Distance value may produce better matching result but will result in inefficiency since a lot of polygons will be selected for second stage matching.

\section{Polygon Matching using Circular Error Bound}

We propose a polygon matching technique based on an intuitive human definition of polygon resemblance. The intuitive definition of similar polygons is as follows. If two polygons are similar (or matched), then each vertex of one polygon is close to its corresponding vertex of another polygon when the two polygons 
are overlapped. The correspondence between vertices is an one-to-one mapping. Therefore, the definition and the technique we proposed only work on polygons that have the same number of vertices. Before the two polygons are overlapped, translation, scaling and rotation are allowed to be performed on the polygons.

Definition 1. A polygon $P$ is represented by an ordered list of vertex coordinates, $P=\left(V_{1}, \ldots, V_{n}\right)$ where $V_{i} \in \mathbb{R}^{2}$ and $n$ is the number of vertices of $P$.

Definition 2. A transformation $T$ is a vector, i.e. $T=\left(t_{x}, t_{y}, s_{x}, s_{y}, \theta\right)$ where $t_{x}$ is translation in $X$ direction, $t_{y}$ is translation in $Y$ direction, $s_{x}$ is the scaling in $X$ direction, $s_{y}$ is the scaling in $Y$ direction, and $\theta$ is the rotation about the origin. $T(Q)$ denotes the polygon (or vertex) obtained by applying $T$ to $Q$.

Definition 3. Given a tolerance vector $E=\left(\epsilon_{1}, \ldots, \epsilon_{n}\right), Q$ is said to be matched with $P$ if there exists a transformation $T$ such that $Q^{\prime}=T(Q)=\left(U_{i}^{\prime}, \ldots, U_{n}^{\prime}\right)$ and $\forall_{1 \leq i \leq n}\left\|V_{i}-U_{i}^{\prime}\right\| \leq \epsilon_{i}$, where $\|\cdot\|$ denotes the Euclidean norm.

Definition 4. Given $V_{i}, \epsilon_{i}$ and $U_{i}$, the $i$ th Circular Error Bound, $C_{i}$, is a circle with $\epsilon_{i}$ as its radius and $\left(V_{i}-U_{i}\right)$ as its center.

Note that Definition 3 assumes we already know the pairing of vertices between the two polygons, i.e. $V_{i}$ should match $U_{i}$.

The polygon matching task is formulated as follows. Given two polygons $P$ and $Q$ with a tolerance vector $E$, the task is to determine whether a transformation $T$ exists such that $Q$ is said to be matched with $P$ under Definition 3 . By Definition 3 , the transformation $T$ is an arbitrary tuple $\left(t_{x}, t_{y}, s_{x}, s_{y}, \theta\right)$. However, in nowadays applications, the transformations in polygon matching task are often restricted to some special cases, for example, translation and (or) scaling only. With restricted transformations, we have efficient solutions for the polygon matching task. In the following sections, we will present the solution for the polygon matching task when (1) only translations are allowed, (2) only translations and uniform scaling in $X, Y$ direction are allowed, and (3) only translations and independent scaling in $X, Y$ direction are allowed.

\subsection{Translation}

Assume that transformation $T$ in Definition 3 is restricted to $T=\left(t_{x}, t_{y}, 1,1,0\right)$.

Proposition 1. Given $P=\left(V_{1}, \ldots, V_{n}\right), Q=\left(U_{1}, \ldots, U_{n}\right), E=\left(\epsilon_{1}, \ldots, \epsilon_{n}\right)$, if the $n$ Circular Error Bounds $C_{1}, \ldots, C_{n}$ of $P$ and $Q$ have common intersection, then $Q$ is matched with $P$.

Proof. Assuming $V_{i}=\left(a_{i}, b_{i}\right)$ and $U_{i}=\left(c_{i}, d_{i}\right)$, by Definition 4, Circular Error Bound $C_{i}$ is a circle with $\epsilon_{i}$ as its radius and $\left(a_{i}-c_{i}, b_{i}-d_{i}\right)$ as its center. If $C_{1}, \ldots, C_{n}$ have common intersection, then for any point $\left(t_{x}, t_{y}\right)$ in the common intersection, the distance between this point and the center of any $C_{i}$ is larger or equal to the radius of $C_{i}$. Figure 2(a) illustrates this idea when both $P$ and $Q$ are triangles. Thus, $\forall_{1 \leq i \leq n},\left[\left(a_{i}-c_{i}\right)-t_{x}\right]^{2}+\left[\left(b_{i}-d_{i}\right)-t_{y}\right]^{2} \leq \epsilon_{i}{ }^{2}$. Re-arranging this equation, we have $\left[a_{i}-\left(c_{i}+t_{x}\right)\right]^{2}+\left[b_{i}-\left(d_{i}+t_{y}\right)\right]^{2} \leq \epsilon_{i}{ }^{2}$ which is equivalent to $\left\|V_{i}-U_{i}^{\prime}\right\| \leq \epsilon_{i}$ where $U_{i}^{\prime}=T\left(U_{i}\right)$ and $T=\left(t_{x}, t_{y}, 1,1,0\right)$. By Definition $3, Q$ is matched with $P$. 


\subsection{Translation and uniform scaling in $X, Y$ direction}

Assume that transformation $T$ in Definition 3 is restricted to $T=\left\langle t_{x}, t_{y}, s, s, 0\right\rangle$. Let $U_{i}=\left(c_{i}, d_{i}\right)$ and apply the scaling transformation $S=(0,0, s, s, 0)$ to $Q$, we have $U_{i}^{\prime}=S\left(U_{i}\right)=\left(s c_{i}, s d_{i}\right)$. Thus, Circular Error Bound $C_{i}$ of $P$ and $Q^{\prime}$, where $V_{i}=\left(a_{i}, b_{i}\right)$, is a circle with $\epsilon_{i}$ as its radius and $\left(a_{i}-s c_{i}, b_{i}-s d_{i}\right)$ as its center. Two Circular Error Bounds $C_{i}$ and $C_{j}$ intersect each other if and only if

$$
\left[\left(a_{i}-s c_{i}\right)-\left(a_{j}-s c_{j}\right)\right]^{2}+\left[\left(b_{i}-s d_{i}\right)-\left(b_{j}-s d_{j}\right)\right]^{2} \leq\left(\epsilon_{i}+\epsilon_{j}\right)^{2}
$$

Re-arranging Equation (1), we have

$$
\begin{array}{r}
{\left[\left(c_{i}-c_{j}\right)^{2}+\left(d_{i}-d_{j}\right)^{2}\right] s^{2}-2\left[\left(a_{i}-a_{j}\right)\left(c_{i}-c_{j}\right)+\left(b_{i}-b_{j}\right)\left(d_{i}-d_{j}\right)\right] s} \\
+\left[\left(c_{i}-c_{j}\right)^{2}+\left(d_{i}-d_{j}\right)^{2}-\left(\epsilon_{i}+\epsilon_{j}\right)^{2}\right] \leq 0
\end{array}
$$

Solving Equation (2), we get a range, $\mathbb{S}_{i j}$, for $s$ that the inequality holds (Figure 2(b)).

Proposition 2. If $\bigcap_{1 \leq i, j \leq n} \mathbb{S}_{i j} \neq \emptyset$, then $Q$ is matched with $P$.

Proof. If $\bigcap_{1 \leq i, j \leq n} \mathbb{S}_{i j} \neq \emptyset$, then $\exists S=(0,0, s, s, 0) \in \bigcap_{1 \leq i, j, \leq n} \mathbb{S}_{i j}$ such that Circular Error Bounds $C_{1}, \ldots, C_{n}$ of $P$ and $Q^{\prime}$ have common intersection, where $Q^{\prime}=S(Q)$. By Proposition $1, Q^{\prime}$ is matched with $P$. Thus, $\exists T=\left(t_{x}, t_{y}, 1,1,0\right)$ such that $\forall_{1 \leq i \leq n}\left\|V_{i}-U_{i}^{\prime \prime}\right\| \leq \epsilon_{i}$ where $U_{i}^{\prime \prime}=T\left(U_{i}^{\prime}\right)$. Therefore, $\exists T^{\prime}=T$ o $S=\left(t_{x}, t_{y}, s, s, 0\right)$ such that $\forall_{1 \leq i \leq n}\left\|V_{i}-U_{i}^{\prime \prime}\right\| \leq \epsilon_{i}$ where $U_{i}^{\prime \prime}=T^{\prime}\left(U_{i}\right)$. By Definition $3, Q$ is matched with $\bar{P}$.

\subsection{Translation and independent scaling in $X, Y$ direction}

Assume that transformation $T$ in Definition 3 is restricted to $T=\left\langle t_{x}, t_{y}, s_{x}, s_{y}, 0\right\rangle$. Let $U_{i}=\left(c_{i}, d_{i}\right)$ and apply the scaling transformation $S=\left(0,0, s_{x}, s_{y}, 0\right)$ to $Q$, we have $U_{i}^{\prime}=S\left(U_{i}\right)=\left(s_{x} c_{i}, s_{y} d_{i}\right)$. Thus, Circular Error Bound $C_{i}$ of $P$ and $Q^{\prime}$, where $V_{i}=\left(a_{i}, b_{i}\right)$, is a circle with $\epsilon_{i}$ as its radius and $\left(a_{i}-s_{x} c_{i}, b_{i}-s_{y} d_{i}\right)$ as its center. Two Circular Error Bounds $C_{i}$ and $C_{j}$ intersect each other if and only if

$$
\left[\left(a_{i}-s_{x} c_{i}\right)-\left(a_{j}-s_{x} c_{j}\right)\right]^{2}+\left[\left(b_{i}-s_{y} d_{i}\right)-\left(b_{j}-s_{y} d_{j}\right)\right]^{2} \leq\left(\epsilon_{i}+\epsilon_{j}\right)^{2}
$$

Re-arranging Equation (3), we have

$$
\left[\left(a_{i}-a_{j}\right)-\left(c_{i}-c_{j}\right) s_{x}\right]^{2}+\left[\left(b_{i}-b_{j}\right)-\left(d_{i}-d_{j}\right) s_{y}\right]^{2} \leq\left(\epsilon_{i}+\epsilon_{j}\right)^{2}
$$

Equation (4) defines an ellipse, $\mathbb{E}_{i j}$, on the $s_{x}-s_{y}$ plane. A point $\left(s_{x}, s_{y}\right)$ in $\mathbb{E}_{i j}$ defines a transformation $S=\left(0,0, s_{x}, s_{y}, 0\right)$ such that when $S$ is applied to $Q$, the Circular Error Bounds $C_{i}$ and $C_{j}$, of $S(Q)$ and $P$, intersect each other (Figure 2(c)).

Proposition 3. If $\forall_{1 \leq i, j \leq n} \mathbb{E}_{i j}$ have common intersection, then $Q$ is matched with $P$.

Proof. If $\forall_{1 \leq i, j \leq n} \mathbb{E}_{i j}$ have common intersection, then for any point $\left(s_{x}, s_{y}\right)$ in the intersection, Circular Error Bounds $C_{1}, \ldots, C_{n}$ of $P$ and $Q^{\prime}$, intersect each other where $Q^{\prime}=S(Q)$ and $S=\left(0,0, s_{x}, s_{y}, 0\right)$. By Proposition 1, $Q^{\prime}$ is matched with $P$. Thus, $\exists T=\left(t_{x}, t_{y}, 1,1,0\right)$ such that $\forall_{1 \leq i \leq n},\left\|V_{i}-U_{i}^{\prime \prime}\right\| \leq \epsilon_{i}$ where $U_{i}^{\prime \prime}=$ $T\left(U_{i}^{\prime}\right)$. Therefore, $\exists T^{\prime}=T \circ S=\left(t_{x}, t_{y}, s_{x}, s_{y}, 0\right)$ such that $\forall_{1 \leq i \leq n},\left\|V_{i}-U_{i}^{\prime \prime}\right\| \leq \epsilon_{i}$ where $U_{i}^{\prime \prime}=T^{\prime}\left(U_{i}\right)$. By Definition $3, Q$ is matched with $P$. 


\section{Minimum Circular Error Bound}

The results presented in Section $3.1,3.2$, and 3.3 only deal with queries of whether a polygon $Q$ is matched with another polygon $P$ subject to some tolerances and under certain transformation restrictions. It is also useful to find out how similar a polygon $Q$ is comparing to another polygon $P$. For example, we may want to rank a list of polygons according to the similarity between these polygons and a target polygon. We propose a translation invariant similarity measure of polygons named Minimum Circular Error Bound (MCEB) based on the Circular Error Bound technique we described above.

Definition 5. The Minimum Circular Error Bound of a polygon $Q=\left(U_{1}, \ldots, U_{n}\right)$ comparing to another polygon $P=\left(V_{1}, \ldots, V_{n}\right)$ is defined as

$$
\xi=\min _{\forall_{t_{x}, t_{y}} T=\left(t_{x}, t_{y}\right)} \max _{1 \leq i \leq n}\left\|V_{i}-T\left(U_{i}\right)\right\|
$$

$\xi$ can be calculated as follows. Let $V_{i}=\left(a_{i}, b_{i}\right)$ and $U_{i}=\left(c_{i}, d_{i}\right)$. Further assume that the tolerance vector $E=\left(\epsilon_{1}, \ldots, \epsilon_{n}\right)$ where $\epsilon_{1}=\cdots=\epsilon_{n}$. The Circular Error Bound $C_{i}$ is a circle with $\epsilon_{i}$ as its radius and $\left(a_{i}-c_{i}, b_{i}-d_{i}\right)$ as its center. If two Circular Error Bounds $C_{i}$ and $C_{j}$ intersect each other, we have

$$
\left[\left(a_{i}-c_{i}\right)-\left(a_{j}-c_{j}\right)\right]^{2}+\left[\left(b_{i}-d_{i}\right)-\left(b_{j}-d_{j}\right)\right]^{2} \leq\left(\epsilon_{i}+\epsilon_{j}\right)^{2}
$$

Since $\epsilon_{i}=\epsilon_{j}$, we denote the value of $\epsilon_{i}$ and $\epsilon_{j}$ as $\epsilon_{i j}$. The minimal value of $\epsilon_{i j}$ that Equation $(5)$ holds is $\epsilon_{i j}=\frac{1}{2} \sqrt{\left[\left(a_{i}-c_{i}\right)-\left(a_{j}-c_{j}\right)\right]^{2}+\left[\left(b_{i}-d_{i}\right)-\left(b_{j}-d_{j}\right)\right]^{2}}$. The MCEB of the two polygons $Q$ and $P$ is $\xi=\max _{1 \leq i, j \leq n} \epsilon_{i j}$, such that for $\epsilon_{1}=\cdots=\epsilon_{n} \geq \xi, \forall_{1 \leq i, j \leq n} C_{i}$ and $C_{j}$ intersect each other. That is, for $\epsilon_{1}=\cdots=\epsilon_{n} \geq \xi$, Circular Error Bounds $C_{1}, \ldots, C_{n}$ of $Q$ and $P$ have common intersection and $Q$ is matched with $P$ under Proposition 1.

\section{$5 \quad$ Experimental Results}

We compare the MCEB method with the Hausdorff Distance method $[3,5]$.

Definition 6. Given two finite point sets $A=\left\{a_{1}, \ldots, a_{n}\right\}$ and $B=\left\{b_{1}, \ldots, b_{m}\right\}$, the Hausdorff Distance is defined as $H(A, B)=\max (h(A, B), h(B, A))$, where $h(A, B)=\max _{a \in A} \min _{b \in B}\|a-b\|$ and $\|\cdot\|$ is some underlying norm on the points of $\mathrm{A}$ and $\mathrm{B}$.

We choose Hausdorff Distance method for comparison since both methods measure polygon similarity based on the distance between polygon vertices. We will compare their similarity ranking results as well as the running time complexity.

\subsection{Similarity Ranking}

We compare the polygon rankings produced by the MCEB and the Hausdorff Distance method. The experiments are conducted as follows. In each experiment, two polygons are selected for generating the input polygons. For example, Figure 3(a) and Figure 3(b) show the two polygons used in one of experiments where 
Figure 3(a) is used as the first polygon and Figure 3(b) is used as the last one. By interpolating these two polygons, 48 intermediate polygons are generated, which gives us 50 polygons in total.

Using this method, we obtain a list of polygons ranked by their relative similarity to the first polygon, which resembles human ranking. We then rank these 50 polygons using the MCEB method and the Hausdorf Distance method accordingly using the first polygon as the target polygon. We use the number of polygons having different relative ranking from the original list as the quality measure of the rankings produced. A small number indicates a good ranking which means that the ranking produced is similar to the original list as well as human visual ranking.

Figure 3(e) and Figure 3(f) show the rankings produced by the MCEB method and the Hausdorff Distance method using Figure 3(a) and Figure 3(b) as the input data. The quality measure of the two rankings are 0 and 9 respectively. Figure $3(\mathrm{~g})$ and Figure $3(\mathrm{~h})$ show the rankings produced by the MCEB method and the Hausdorff Distance method using Figure 3(c) and Figure 3(d) as the input data. The quality measure of the two rankings are 0 and 13 respectively. In these two experiments, the MCEB method produces better rankings than the Hausdorff Distance method.

\subsection{Running Time Complexity}

The computational complexity of the Hausdorff Distance is $O\left(n^{2}\right)$ and it is $O\left(n^{2}\right)$ for the MCEB method, if the correspondence of vertices is known, or $O\left(n^{3}\right)$ if the correspondence of vertices is unknown since we have to exhaust the $n$ possible correspondences in order to find out the overall MCEB for a $n$-gon. However, note that the MCEB method gives similarity measure between two polygons under the optimal translation, but the Hausdorff Distance method does not. We may want to use the Hausdorff Distance under optimal translation for ranking polygons instead of the original Hausdorff Distance in order to produce better ranking of polygons. The computational complexity for optimal Hausdorff Distance under translation is $O\left(n^{4} \log ^{3}\left(n^{2}\right)\right)$ [1], which is much larger than that of the MCEB method.

Table 1 shows the average query processing time of the MCEB method, the Hausdorff Distance method and the MRAM method we that proposed in $[12,11]$. The experiments are conducted using the simple system we described in [12]. There are 9000 polygons in each testing database and each database is consisted of polygons with a specific number of sides, from 3 to 8 . The table shows that MCEB has an average query processing time less than the Hausdorff Distance method, while the MRAM has the smallest average query processing time among the three methods.

\section{Conclusion}

In this paper, we introduce an extension to the two-stage framework for polygon retrieval we proposed to augment the framework with systematic control on search space pruning. We also propose a new polygon matching technique using Circular Error Bound (CEB) method which is based on an intuitive human idea of polygon resemblance. Based on $\mathrm{CEB}$, we propose a translation invariant 
similarity measure of polygons named Minimum Circular Error Bound (MCEB). We find that the MCEB method gives rankings of polygons similar to human rankings and it is more efficient than the Hausdorff Distance method.

Right now, the CEB method only handles polygon matching under translation and scaling. A natural extension to the CEB method is to incorporate rotation. Another possible extension to our work is to enhance the translation invariant $M C E B$ method such that it is scaling invariant as well.

\section{References}

1. P. K. Agarwa, M. Sharir, and S. Toledo. Applications of Parametric Searching in Geometric Optimization. Proc. of the 3rd Annual ACM-SIAM Symposium on Discrete Algorthms, pages pp. 72-82, 1992.

2. B. Bhavnagri. A Method for Representing Shape Based on an Equivalent Relat ion on Polygons. Pattern Recognition, Vol. 27(No. 2):pp. 247-260, 1994.

3. Daniel P. Huttenlocher and William J. Rucklidge. A Multi-Resolution Technique for Comparing Images Using the Hausdorff Distance. Department of Computer Science, Cornell University, Technical Report TR92-1328, 1992.

4. H. V. Jagadish. A Retrieval Technique for Similar Shapes. Proc. of the ACM SIGMOD Int. Conf. on the Management of Data, pages pp. 208-217, May 1991.

5. Helmut Alt, Bernd Behrebds, and Johnnes Blömer. Approximate Matching of Polygonal Shapes. Annals of Mathematics and Artificial Intelligence, Vol. 13:pp. 251-265, 1995

6. Jia Guu Leu. Computing a Shape's Moments from its Boundary. Pattern Recognttion, Vol. 24(No. 10):pp. 949-957, 1991.

7. Jonathan Ashley, Ron Barber, Myron Flickner, James Hafner, Denis Lee, Wayne Niblack, and Dragutin Petkovic. Automatic and Semi-Automatic Methods for Image Annotation and Retrieval in QBIC. SPIE, Vol. 2420:pp. 24-35, 1995.

8. I. King and T. K. Lau. A Feature-Bassed Image Retrieval Database for the Fashion, Textile, and Clothing Industry in Hong Kong. Proc. of Internatzonal Symposium Multi-Technology Information Processing '96, pages pp. 233-240, 1996.

9. Pedro Cox, Henri Maitre, Michel Minoux, and Celso Ribeiro. Optimal Matching of Convex Polygons. Pattern Recognition Letters, Vol. 9:pp. 327-334, 1989

10. Rajiv Mehrotra and James E. Gary. Feature-Based Retrieval of Similar Shape. Proc. 9th Int. Conf. on Data Engineering, pages pp. 108-115, 1993.

11. L. H. Tung, I. King, P. F. Fung, and W. S. Lee. A Two-Stage Framework for Efficient Simple Polygon Retrieval in Image Databases, journal = Proc. of International Symposium MultiTechnology Information Processing '96. 1996

12. L. H. Tung, I. King, P. F. Fung, and W. S. Lee. Two-Stage Polygon Representation for Efficient Shape Retrieval in Image Databases. Proc. of the 1st International Workshop on Image Databases and Multimedia Search, 1996.

13. W. Niblack, R. Barber, W. Equitz, M. Flickner, E. Glasman, D. Petkovic, and P. Yanker. The QBIC Project: Querying Images By Content Using Color, Texture, and Shape. SPIE, Vol. 1908:pp. 173-187, 1993 .

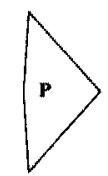

$S B S D=0 \times 00$

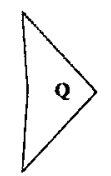

SBSD $=0001$

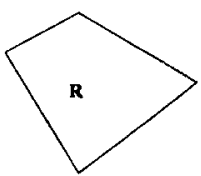

SBSD $=0000$

Fig. 1. The problem of the two-stage framework 


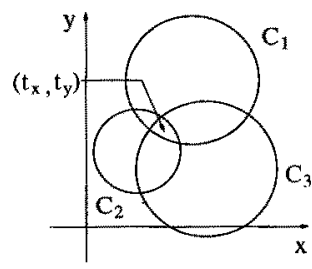

(a)

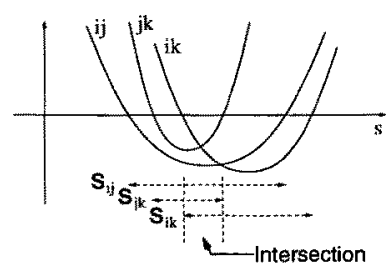

(b)

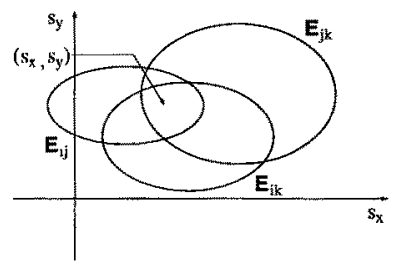

(c)

Fig. 2. (a) Intersection of Circular Error Bounds (b) $\mathbb{S}_{i j}$ and their intersection (c) $\mathbb{E}_{i j}$ and their intersection

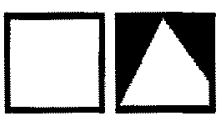

(a)

(b)

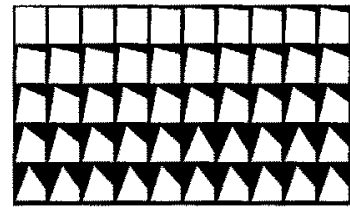

(f)

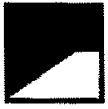

(c)

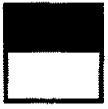

(d)

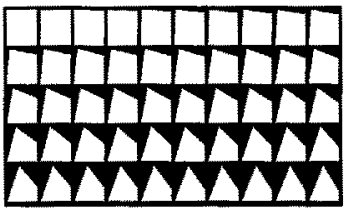

(e)

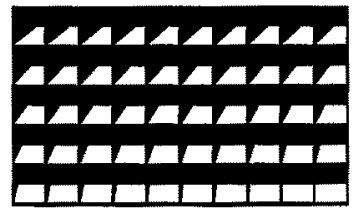

(g)

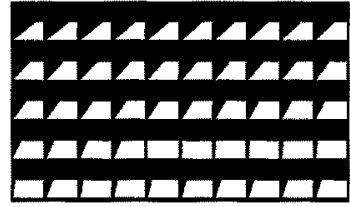

(h)

Fig. 3. Similar ranking experiments

Table 1. Experiment results

\begin{tabular}{|c||c|c|c|c|}
\hline N-gon & Number of polygons & MAAM & MCEB & Haugdorfi Distance \\
\hline 3 & 9000 & $2.04 \mathrm{sec}$ & $3.24 \mathrm{sec}$ & $3.40 \mathrm{sec}$ \\
\hline 4 & 9000 & $1.97 \mathrm{sec}$ & $2.40 \mathrm{sec}$ & $2.62 \mathrm{sec}$ \\
\hline 5 & 9000 & $1.74 \mathrm{sec}$ & $2.00 \mathrm{sec}$ & $2.21 \mathrm{sec}$ \\
\hline 6 & 9000 & $1.64 \mathrm{sec}$ & $1.78 \mathrm{sec}$ & $1.87 \mathrm{sec}$ \\
\hline 7 & 9000 & $1.62 \mathrm{sec}$ & $1.68 \mathrm{sec}$ & $1.72 \mathrm{sec}$ \\
\hline 8 & 9000 & $1.63 \mathrm{sec}$ & $1.67 \mathrm{sec}$ & $1.68 \mathrm{sec}$ \\
\hline
\end{tabular}

